\title{
Extending the weak order on Coxeter groups
}

\author{
François Viard $\|^{\dagger}$ \\ ${ }^{1}$ Université Lyon 1, Institut Camille Jordan, UMR 5208 du CNRS, 69622 Villeurbanne, France
}

\begin{abstract}
We introduce a new family of complete lattices, arising from a digraph together with a valuation on its vertices and generalizing a previous construction of the author. We then apply this to the study of two long-standing conjectures of Dyer, and we provide a description of the Tamari lattice with this theory.

Résumé. On introduit une nouvelle famille de treillis complets, définis à partir de graphes orientés munis d'une valuation sur leurs sommets, qui généralisent une précédente construction de l'auteur. On applique ensuite cette construction à l'étude de deux conjectures de Dyer, et on fournit une description du treillis de Tamari à l'aide de notre théorie.
\end{abstract}

Keywords. Digraphs, Coxeter groups, weak order, root systems, Tamari lattice

\section{Introduction}

This abstract presents some developments concerning the objects introduced in [17]. More precisely, in this abstract we develop a new theoretic framework based on digraphs for the study of weak order on any Coxeter group, and we apply this to the study of various associated questions.

Here, the first problem we focus on is a set of several conjectures of Dyer about an extension of the weak order, which would allow us to extend combinatorial and geometrical properties usually confined to finite Coxeter groups to all Coxeter groups. Even if these conjectures first appear more than 20 years ago (see [2] and [3]), little is known about them. However, they recently become the object of intensive studies, motivating the study of asymptotical behaviour of roots systems (see, for instance, [6] and [8]), of abstract constructions (see [4] and [5]) and of geometrical properties of root systems (see [7] and [9]).

One of the possible motivations for the study of Dyer's conjectures is the study of Cambrian lattices (see [11] and [13]). Cambrian lattices are quotients of the weak order of any finite Coxeter groups, which are related to combinatorics of cluster algebras. In particular, the Hasse diagram of a Cambrian lattice is isomorphic to the exchange graph of the cluster algebra associated with the considered Coxeter group (see [12] and [15]). Their definition can be extended to any Coxeter group (see [14]), but the correspondence with cluster algebra is only partial. For example, the Hasse diagram of the resulting poset (called Cambrian semi-lattice) is isomorphic to a sub-graph of the corresponding cluster algebra (see [15]). Even if in the case of affine Coxeter groups Reading and Speyer showed in [16] how to extend these Cambrian semi-lattices in order to obtain all the expected information about cluster algebras, this

\footnotetext{
$\dagger$ Email: viardemath.univ-lyonl.fr. 
problem remains open in general. However, Dyer's conjectures provide a candidate for an extension of the weak order on any Coxeter group. Thus, it is natural to look for a way to extend the construction of Cambrian semi-lattice to these extensions, hoping that one could recover more information about cluster algebras from it. This constitutes the second problem whose study is started here, describing Tamari lattice using our theory

\section{Previous results (acyclic case)}

In this section, we briefly detail the results from [17]. The fundamental object of this abstract is what we call a valued digraph $\mathcal{G}=(G, \theta)$, where $G$ is a simple digraph (not necessarily finite) with set of vertices $\mathcal{V}(G)$ and set of arcs $E(\mathcal{G})$, and $\theta$ is a function from $\mathcal{V}(G)$ to $\mathbb{N}$, satisfying the following inequality:

$$
\text { for all } z \in \mathcal{V}(G), 0 \leq \theta(z) \leq d^{+}(z),
$$

where $d^{+}(z)$ denotes the out-degree of the vertex $z$ (i.e. the number of arcs having $z$ as starting point). In this section, we restrict our study to the case where the underlying digraph is acyclic.

We then define an algorithm which will be used to generate sequences of elements of $\mathcal{V}(G)$, called peeling sequences of $G$, by peeling the digraph vertex by vertex with respect to the constraint given by the valuation $\theta$. More precisely, we first chose an erasable vertex $z$ of $\mathcal{V}(G)$, that is a vertex such that:

- $\theta(z)=0$

- for all $y \in \mathcal{V}(G)$, if there is an arc from $y$ to $z$ in $G$, then $\theta(y)>0$.

We then peel this vertex, that is:

1. we decrease by one the valuation on each vertex $y$ such that there is an arc from $y$ to $z$;

2. we erase the vertex $z$ and the arcs having $z$ as starting or ending point.

What we obtain after this operation is again a valued digraph, so that we can iterate this process. An iteration of this peeling process is depicted in Figure 1 .

We denote by $P S(\mathcal{G})$ the set of all the peeling sequences, and we consider the set $I S(\mathcal{G})$ of all the initial sections of the elements of $P S(\mathcal{G})$, that are the sets of the form $\left\{z_{1}, \ldots, z_{k}\right\}$ where the sequence $\left[z_{1}, \ldots, z_{k}\right]$ is the beginning of a peeling sequence of $\mathcal{G}$. We then show that the poset $(I S(\mathcal{G}), \subseteq)$ has many properties similar to those of the weak order. Namely, we prove that the poset $(I S(\mathcal{G}), \subseteq)$ is a graded complete meet semi-lattice in general, a graded complete lattice when the underlying digraph is finite, and that its Möbius function takes values into the set $\{0,1,-1\}$. Eventually, we prove that the weak order on Coxeter groups of type $A, B, D, E_{6}, E_{7} E_{8}$ and $\widetilde{A}$ can be described within this theory, constructing explicitly an associated acyclic valued digraph (we refer the interested reader to [17] for a detailed construction of the valued digraphs). Let us explain a little bit the method we follow to prove that the weak order on $A_{n-1}$ can be described using a valued digraph. It is well known that $A_{n-1}$ can be identified with the symmetric group $S_{n}$, and there is a canonical set associated with each permutation $\sigma \in S_{n}$ : its inversion set, defined by

$$
\operatorname{Inv}(\sigma):=\left\{(a, b) \in \mathbb{N}^{2} \mid 1 \leq a<b \leq n \text { and } \sigma^{-1}(a)>\sigma^{-1}(b)\right\} .
$$



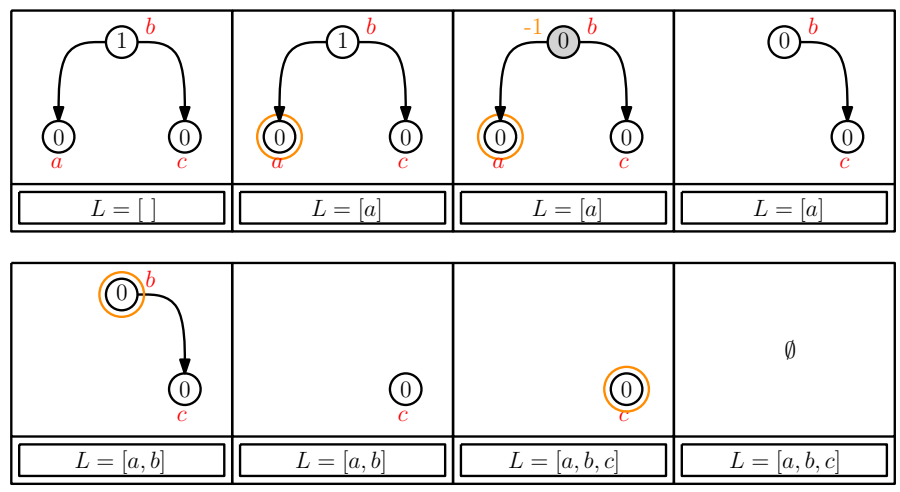

Fig. 1: Peeling process in action. The resulting sequence $L$ is what we call a peeling sequence of $\mathcal{G}$.

We then have the following classical property:

$$
\text { for all } \sigma, \omega \in S_{n}, \sigma \leq_{R} \omega \text { if and only if } \operatorname{Inv}(\sigma) \subseteq \operatorname{Inv}(\omega) \text {, }
$$

where $\leq_{R}$ denotes the (right) weak order on $S_{n}$. We then construct a valued digraph $\mathcal{A}$ whose vertices are indexed by all pairs of integers $(a, b)$ such that $1 \leq a<b \leq n$, and such that $\mathcal{A}$ satisfies the following property: $I S(\mathcal{A})=\left\{\operatorname{Inv}(\sigma) \mid \sigma \in S_{n}\right\}$, implying immediately that $(I S(\mathcal{A}), \subseteq)$ and $\left(A_{n-1}, \leq_{R}\right)$ are isomorphic.

\section{A more general construction}

It is natural to ask if these previous results can be generalized to any Coxeter group. However, in this larger arena many difficulties appear, and to overcome these we have to change our point of view on the considered objects. In general, we do not have an interpretation of Coxeter groups as a group of permutations (or similar objects), but we have a general notion of inversion set thanks to root systems. Root systems are geometrical objects allowing us to see any Coxeter group as a reflection group, but generally not in an Euclidean space. More precisely, a root system of a Coxeter group $W$ of rank $n$ is a subset $\Phi$ of the vector space $\mathbb{R}^{n}$ on which $W$ acts and allowing us to interpret geometrically many properties of $W$. In particular, there exists a partition of $\Phi$ into two subsets $\Phi^{+}$and $\Phi^{-}$, respectively called the sets of positive and negative roots, from which we can define a notion of inversion set: for all $w \in W$, we set

$$
\operatorname{Inv}(w):=\Phi^{+} \cap w\left(\Phi^{-}\right) .
$$

It is shown in [1] that for all $w^{\prime} \in W, w$ is smaller than $w$ for the weak order if and only if $\operatorname{Inv}(w) \subseteq$ $\operatorname{Inv}\left(w^{\prime}\right)$.

Then, the idea is to construct a valued digraph $\mathcal{G}$ whose vertices are the elements of $\Phi^{+}$and such that $I S(\mathcal{G})$ is made exactly of the inversion sets of the elements of $W$. However, two major difficulties appear: there is no canonical choice for a valued digraph in the case of dihedral groups (many valued digraphs work); when we try to generalize the construction to the case of any Coxeter group, the resulting digraph is generally not acyclic, so that the theory developed in [17] does not apply. These two remarks lead us to the developments presented in the following sections. 


\subsection{The general theory and first conjecture of Dyer}

We begin with generalizing the construction presented in Section 2, allowing us to study the case where the valued digraph is not acyclic. This new construction is a natural consequence of the content of [17]. Indeed, in order to simplify some proofs we introduced a characterization of the elements of $I S(\mathcal{G})$ (see [17. Prop. 3.1]), which does not rely on peeling sequences and does not rely on the properties of the digraph (such as acyclicity). Therefore, we can take this characterization as a definition of $I S(\mathcal{G})$ and remove the conditions on the digraph. More precisely, we have the following definition.

Definition 3.1 Let $\mathcal{G}=(G, \theta)$ be a pair of a digraph $G$ and a valuation $\theta$ satisfying $0 \leq \theta(z) \leq d^{+}(z)$ for all $z \in \mathcal{V}(G)$. We denote by $I S(\mathcal{G})$ the set defined by:

$$
I S(\mathcal{G}):=\left\{\begin{array}{l|l}
A \subseteq \mathcal{V}(\mathcal{G}) & \begin{array}{l}
\theta(z) \leq d_{A}^{+}(z) \text { for all } z \in A \\
\theta(z) \geq d_{A}^{+}(z) \text { for all } z \in \mathcal{V}(\mathcal{G}) \backslash A
\end{array}
\end{array}\right\},
$$

where $d_{A}^{+}(z)$ is the number of arcs in $G$ having $z$ as starting point and an element of $A$ as ending point.

This definition generalizes the one proposed in Section 2 , but note that a subtlety appears. When the digraph is infinite, simple and acyclic, then, with this definition, $I S(\mathcal{G})$ contains sets being infinite (for example, $\mathcal{V}(G)$ is always in $I S(\mathcal{G})$ ), and these sets can not be defined using initial sections of peeling sequences. In this more general framework, we have the following theorem.

Theorem 3.2 For all valued digraph $\mathcal{G}$, the poset $(I S(\mathcal{G}), \subseteq)$ is a complete lattice.

Proof: First, note that both $\mathcal{V}(\mathcal{G})$ and $\emptyset$ are in $I S(\mathcal{G})$. Thus, $(I S(\mathcal{G}), \subseteq)$ is bounded, and it is enough to prove that it is a complete join semi-lattice.

We now construct explicitly the join of any subset of $I S(\mathcal{G})$. For that purpose, fix $X \subseteq I S(\mathcal{G})$ and denote by $J_{0}$ the set $\bigcup_{B \in X} B$, we then define recursively a sequence $\left(J_{i}\right)_{i \geq 0}$ as follows:

$$
\forall i \geq 0, J_{i+1}=J_{i} \cup\left\{z \in \mathcal{V}(\mathcal{G}) \backslash J_{i} \mid \theta(z)<d_{J_{i}}^{+}(z)\right\} .
$$

Obviously, we have $J_{0} \subseteq J_{1} \subseteq J_{2} \subseteq \ldots$, and we denote by $J_{\infty}=\cup_{i \geq 0} J_{i}$ the limit of these sets. We now show that $J_{\infty}$ is in $I S(\overline{\mathcal{G}})$. Let $z \in \overline{\mathcal{V}}(\mathcal{G})$, we have three cases.

- If $z \in J_{0}$, then there exists $B \in X$ such that $z \in B$, so that $\theta(z) \leq d_{B}^{+}(z)$, but $B \subseteq J_{0} \subseteq J_{\infty}$, hence $\theta(z) \leq d_{J_{\infty}}^{+}(z)$.

- If $z \in J_{\infty} \backslash J_{0}$, then there exists $i \geq 0$ such that $z \in J_{i+1} \backslash J_{i}$, hence $\theta(z)<d_{J_{i}}^{+}(z) \leq d_{J_{\infty}}^{+}(z)$.

- If $z \in \mathcal{V}(\mathcal{G}) \backslash J_{\infty}$, then for all $i \geq 0$ we have $\theta(z) \geq d_{J_{i}}^{+}(z)$. Thus, if we denote by $C_{i}$ the set $\left\{(z, y) \in E(\mathcal{G}) \mid y \in J_{i}\right\}$ where the arcs are counted with multiplicity, then we have $C_{0} \subseteq C_{1} \subseteq$ $C_{2} \subseteq \ldots$ and $\left|C_{i}\right|=d_{J_{i}}^{+}(z) \leq \theta(z)$ for all $i \geq 0$. Therefore, the sequence $\left(C_{i}\right)_{i \geq 0}$ is stationary at a given rank, i.e. there exists $N \geq 0$ such that for all $m \geq N, C_{m}=C_{N}$. Thus, we have $d_{J_{\infty}}^{+}(z)=d_{J_{N}}^{+}(z) \leq \theta(z)$. 
It follows that $J_{\infty}$ is in $I S(\mathcal{G})$.

Finally, we show that $J_{\infty}$ is the join of $X$. Let $A$ be an upper bound of $X$, we show by induction on $i$ that $J_{i} \subseteq A$ for all $i \geq 0$. Since $A$ is an upper bound of $X$, we have $J_{0} \subseteq A$. Let $i \geq 0$ be such that the property is true and consider $z \in J_{i+1} \backslash J_{i}$, we have $\theta(z)<d_{J_{i}}^{+}(z) \leq d_{A}^{+}(z)$ by induction hypothesis. Therefore, $z \in A$, so that $J_{i+1} \subseteq A$. Thus, we have $J_{\infty} \subseteq A$, which implies that $J_{\infty}$ is the join of $X$ in $(I S(\mathcal{G}), \subseteq)$, and this ends the proof.

In particular, Theorem 3.2 provides a natural embedding of posets of Section 2 into complete lattices (when the underlying digraphs are infinite). This last point is particularly important for the sequel of this abstract. Indeed, Dyer conjectured in [2] and [3] that it is possible to extend the weak order on any Coxeter group into a complete lattice. This conjecture uses the notion of bi-closed sets of $\Phi^{+}$. We say that a set $A \subseteq \Phi^{+}$is closed if and only if for all $\alpha, \beta, \gamma \in \Phi^{+}$such that $\gamma=a \alpha+b \beta$, with $a>0$ and $b>0$ we have that: if $\alpha, \beta \in A$, then $\gamma \in A$.

We say that $A$ is bi-closed if and only if both $A$ and $\Phi^{+} \backslash A$ are closed, and we denote by $\mathcal{B}\left(\Phi^{+}\right)$the set of bi-closed sets of $\Phi^{+}$. It is known that finite bi-closed sets are exactly the inversion sets of the elements of $W$ (see, for instance, [10]). Thus, the poset $\left(\mathcal{B}\left(\Phi^{+}\right), \subseteq\right.$ ) is an extension of the weak order on $W$, and Dyer's conjecture says that this poset is a complete lattice. We represent in Figure 2 the poset $\left(\mathcal{B}\left(\Phi^{+}\right), \subseteq\right)$ obtained considering bi-closed sets of a root system of the infinite dihedral group. Therefore, the idea is to
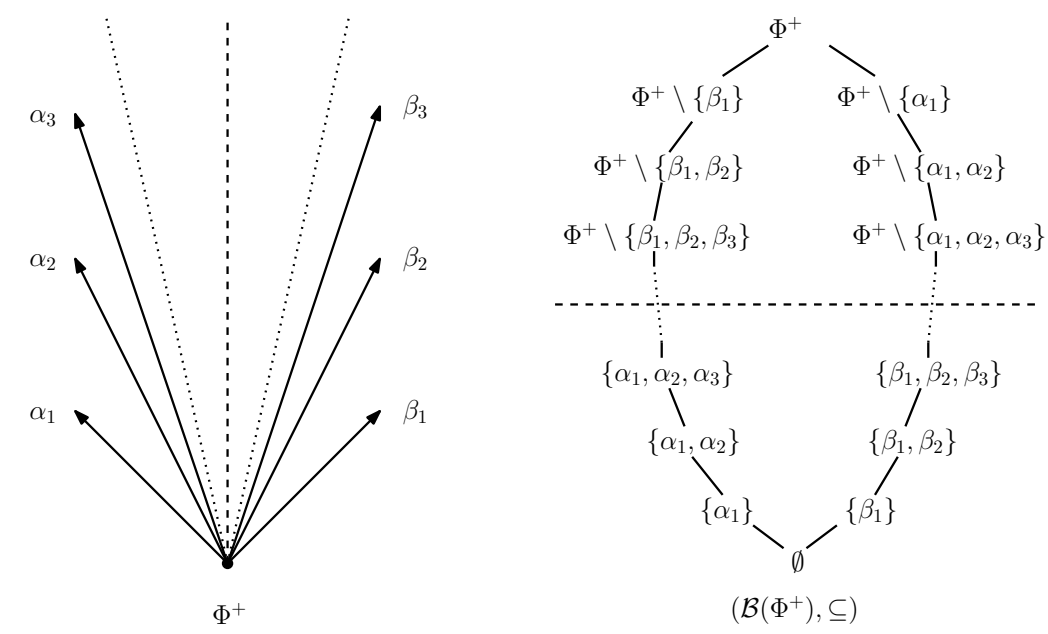

Fig. 2: On the right, the poset $\left(\mathcal{B}\left(\Phi^{+}\right), \subseteq\right)$ obtained considering a root system of the infinite dihedral group (on the left).

look for a valued digraph $\mathcal{G}$ having $\Phi^{+}$as set of vertices and such that $I S(\mathcal{G})=\mathcal{B}\left(\Phi^{+}\right)$. In the case of the dihedral group, this new constraint restricts considerably the available choices and leads us to introduce a new family of valued digraph that we call scaffoldings.

Definition 3.3 Let $a$ and $b$ be two injective sequences on two disjoint sets. A digraph $G$ is called a scaffolding made of $a$ and $b$ if and only if there exists a sequence $c$ such that:

- $c$ is a shuffle of a and $b$; 
- $\mathcal{V}(\mathcal{G})=\operatorname{Im}(a) \sqcup \operatorname{Im}(b)=\operatorname{Im}(c)$;

- $\left\{c_{1}, c_{2}\right\}=\left\{a_{1}, b_{1}\right\}$;

- the out-degree of $c_{1}$ and $c_{2}$ is 0 ;

- for all $j \geq 3$, if we denote by $j_{a}$ (resp. $j_{b}$ ) the maximal integer such that $j_{a}<j\left(\right.$ resp. $j_{b}<j$ ) and $c_{j_{a}} \in \operatorname{Im}(A)\left(\right.$ resp. $\left.c_{j_{b}} \in \operatorname{Im}(b)\right)$, then $\left(c_{j}, c_{j_{a}}\right)$ and $\left(c_{j}, c_{j_{b}}\right)$ are the only arcs having $c_{j}$ as starting point in $G$.

By definition, it is clear that any vertex of a scaffolding is either of out-degree 0 , or of out-degree 2 . Thus, there is a canonical valued digraph associated with any scaffolding, where the valuation on each vertex is given by its out-degree divided by 2 .
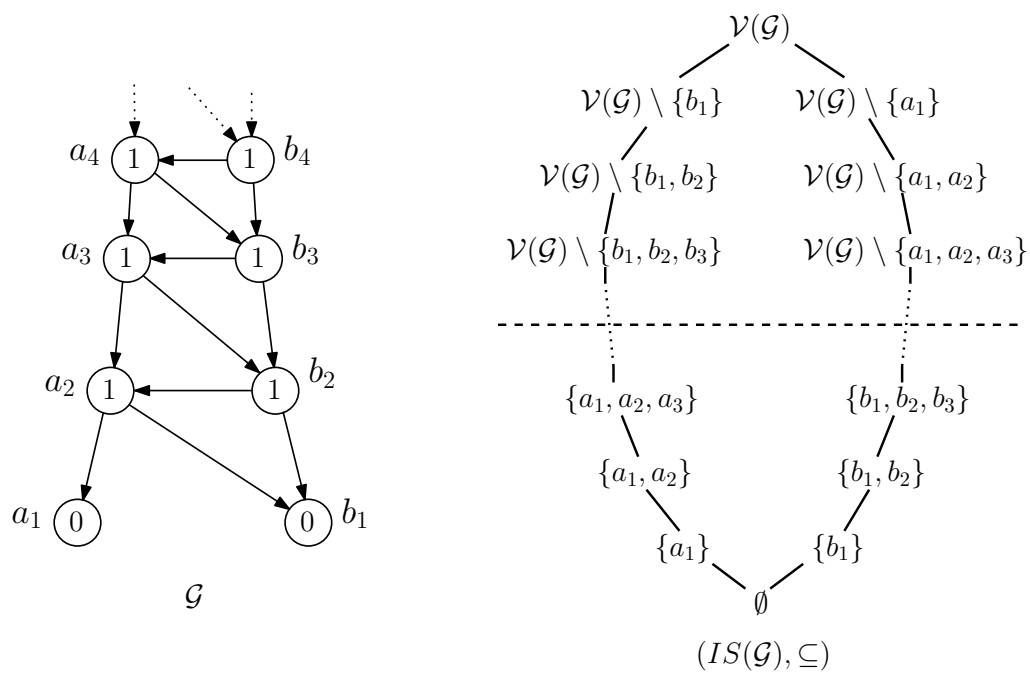

Fig. 3: On the left, the valued digraph associated with the scaffolding $\mathcal{G}$ comming from the sequence $c=$ $\left[a_{1}, b_{1}, a_{2}, b_{2}, \ldots\right]$; on the right, the resulting lattice $(I S(\mathcal{G}), \subseteq)$.

We see on Figure 3 that the lattice obtained with this scaffolding and the poset of the bi-closed sets of the infinite dihedral group are isomorphic, identifying $\alpha_{i}$ with $a_{i}$ and $\beta_{i}$ with $b_{i}$ (this remains true for most scaffoldings, see [18, Chapter 4]). Using this information, we can use scaffoldings (more precisely, we glue scaffoldings together with respect to some constraint given by the geometry of the root system) in order to construct a family of valued digraph that we call well-assembled on $\Phi^{+}$(see [18, Chapter 4] for more details). We then show that for all well-assembled on $\Phi^{+}$valued digraph $\mathcal{G}$, we have $\mathcal{B}\left(\Phi^{+}\right) \subseteq$ $I S(\mathcal{G})$ and for all $A \in I S(\mathcal{G})$, we have $\Phi^{+} \backslash A \in I S(\mathcal{G})$. Thus, we have an embedding of $\left(\mathcal{B}\left(\Phi^{+}\right), \subseteq\right)$ into a complete lattice (more precisely, it is an ortho-lattice, but we will not discuss it here).

\subsection{Projective valued digraphs and the second conjecture of Dyer}

One of the main difficulties appearing at this stage is the infinite character of the considered valued digraph. Indeed, it is quite technical to make tests in order to identify the elements of $I S(\mathcal{G})$, and this 
problem leads us to the definition of a new family of valued digraph, namely projective valued digraphs. A natural starting point is to consider digraphs (we will consider valued digraphs a little bit after) that can be seen as a limit of a sequence of finite acyclic digraphs. Indeed, in the case of finite acyclic valued digraph, we can use the peeling process in order to efficiently identify all the elements of $I S(\mathcal{G})$, and it is natural to see if these kind of tools pass to a well-chosen family of limit digraph. There are many natural way to define a notion of limit of a sequence of digraphs, and the one we will consider here is perhaps one of the simplest: let $\left(G_{i}\right)_{i \geq 1}$ be a sequence of digraphs such that:

1. $G_{1}$ is finite, simple and acyclic;

2. $\mathcal{V}\left(G_{i+1}\right)$ is obtained from $\mathcal{V}\left(G_{i}\right)$ by adding a new vertex $z_{i+1}$ to it;

3. $E\left(G_{i+1}\right)$ is obtained from $E\left(G_{i}\right)$ by adding some new arcs to it, each one having $z_{i+1}$ as starting point (notice that we do not allow to add arcs having $z_{i+1}$ as ending point, and that we do not allow multiple arcs).

Such a sequence clearly defines what corresponds to the intuitive notion of "limit of a sequence of digraphs", that is the digraph $G_{\infty}$ such that

$$
\mathcal{V}\left(G_{\infty}\right)=\bigcup_{i \geq 1} \mathcal{V}\left(G_{i}\right) \text { and } E\left(G_{\infty}\right)=\bigcup_{i \geq 1} E\left(G_{i}\right)
$$

On Figure 4 we represent the beginning of such a sequence of digraphs. With these conditions, the

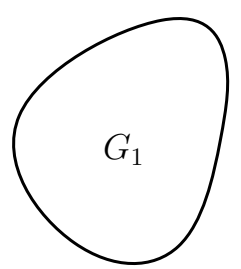

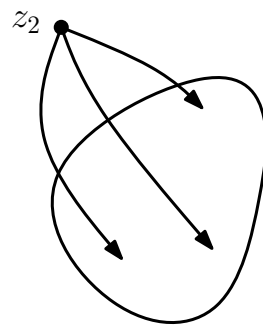

$G_{2}$

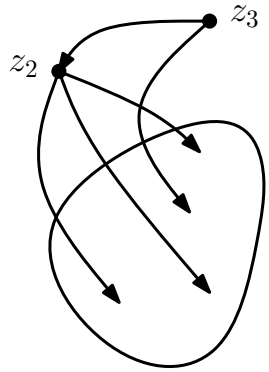

$G_{3}$

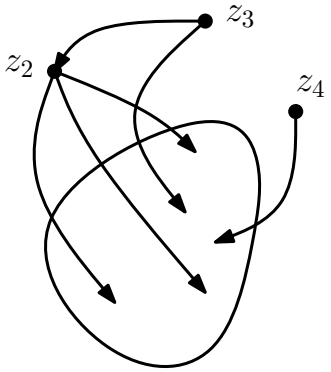

$G_{4}$

Fig. 4: The beginning of a sequence of digraph defining a limit according to our definition.

sequence of digraph is made of finite acyclic digraphs, so that $G_{\infty}$ is indeed a limit of simple acyclic digraphs. However, the main advantage of this definition lies on a technical point, related to our theory of valued digraphs. That is, consider A a subset of $\mathcal{V}\left(G_{j}\right)$ and $z \in \mathcal{V}\left(G_{i}\right)$, with $i<j$. Then, we have

$$
d_{A}^{+}\left(G_{j}, z\right)=d_{A \cap \mathcal{V}\left(G_{i}\right)}^{+}\left(G_{j}, z\right)=d_{A \cap \mathcal{V}\left(G_{i}\right)}^{+}\left(G_{i}, z\right)
$$

Where $d_{A}^{+}(G, z)$ denotes the number of arcs of $G$ having $z$ as starting point and an element of $A$ as ending point. Indeed, by definition of the sequence $\left(G_{i}\right)_{i \geq 1}$ there is no $\operatorname{arc}$ in $G_{j}$ having $z$ as starting point and 
an element of $\mathcal{V}\left(G_{j}\right) \backslash \mathcal{V}\left(G_{i}\right)$ as ending point. Equation (1) has two immediate consequences, which are fundamental in what follows.

If we consider a valuation $\theta: \mathcal{V}\left(G_{j}\right) \rightarrow \mathbb{N}$ such that $\mathcal{G}_{j}=\left(G_{j}, \theta\right)$ is a valued digraph, then for all $z \in \mathcal{V}\left(G_{i}\right)$ we have:

$$
0 \leq \theta(z) \leq d_{\mathcal{V}\left(G_{j}\right)}^{+}\left(G_{j}, z\right) \Longrightarrow 0 \leq \theta(z) \leq d_{\mathcal{V}\left(G_{i}\right)}^{+}\left(G_{i}, z\right),
$$

so that $\mathcal{G}_{i}=\left(G_{i}, \theta\right)$ is also a valued digraph. More interesting, for all $A \subseteq \mathcal{V}\left(\mathcal{G}_{j}\right)$, we have

$$
A \in I S\left(\mathcal{G}_{j}\right) \Longleftrightarrow\left\{\begin{array}{l}
\theta(z) \leq d_{A}^{+}\left(G_{j}, z\right) \text { for all } z \in A \\
\theta(z) \geq d_{A}^{+}\left(G_{j}, z\right) \text { for all } z \in \mathcal{V}\left(G_{j}\right) \backslash A
\end{array}\right.
$$

so that we have

$$
A \in I S\left(\mathcal{G}_{j}\right) \Longrightarrow\left\{\begin{array}{l}
\theta(z) \leq d_{A \cap \mathcal{V}\left(G_{i}\right)}^{+}\left(G_{i}, z\right) \text { for all } z \in A \cap \mathcal{V}\left(G_{i}\right) \\
\theta(z) \geq d_{A \cap \mathcal{V}\left(G_{i}\right)}^{+}\left(G_{i}, z\right) \text { for all } z \in \mathcal{V}\left(G_{i}\right) \backslash A
\end{array}\right.
$$

and finally, by definition of $I S\left(\mathcal{G}_{i}\right)$ we have

$$
A \in I S\left(\mathcal{G}_{j}\right) \Longrightarrow \mathcal{V}\left(G_{i}\right) \cap A \in I S\left(\mathcal{G}_{i}\right) .
$$

In other words, we can project $I S\left(\mathcal{G}_{j}\right)$ on $I S\left(\mathcal{G}_{i}\right)$ (and this projection is surjective, see [18, Section 4.2]) and the family $\left(I S\left(\mathcal{G}_{j}\right)\right)_{i \geq 1}$ is what we call a projective system for a well-chosen set of projections (see the definition below).

Definition 3.4 $A$ projective system is a family $\left(A_{i}, p_{j, i}\right)_{1 \leq i<j}$, where the indices are taken in $\mathbb{N} \cup\{\infty\}$, $A_{i}$ is a set and $p_{j, i}$ is a function from $A_{j}$ to $A_{i}$ such that

$$
p_{k, i} \text { o } p_{j, k}=p_{j, i} \text { for all } i<k<j .
$$

Consequently, for any element $A$ in $I S\left(G_{\infty}\right)$ (a limit valued digraph, once we fixed a valuation), there exists a sequence of elements $\left(A_{i}\right)_{i \geq 1}$ obtained by projecting $A$ on each $I S\left(G_{i}\right)$, each one of these $A_{i}$ being finite. This property is in fact way more important than it probably appear to the reader, and makes the connection between this construction and combinatorics. Indeed, we can show that the elements of $I S\left(\mathcal{G}_{\infty}\right)$ are completely determined by their sequence of projections, and we can study the lattice structure of $\left(I S\left(\mathcal{G}_{\infty}\right), \subseteq\right)$ using the sequence of finite lattices $\left(I S\left(G_{i}\right), \subseteq\right)$, allowing us to make effective tests despite the infinite nature of the considered objects. For more details, we refer the reader to [18, Section 4.2].

From now on, a projective valued digraph will be a valued digraph whose underlying digraph is a limit of finite simple acyclic digraphs (as defined earlier), and we will usually denote by $\mathcal{G}_{\infty}$ a projective valued digraph and by $\left(G_{i}\right)_{i \geq 1}$ its associated sequence of finite acyclic valued digraphs. An interesting point about projective valued digraphs is that they naturally lead to consider another conjecture of Dyer (see [3]), related to the structure of maximal chains of $\left(\mathcal{B}\left(\Phi^{+}\right), \subseteq\right)$ and using the notion of reflection orderings of $\Phi^{+}$. A reflection ordering is a total order $I=\left(\Phi^{+}, \preceq\right)$ of $\Phi^{+}$, such that for all $\alpha, \beta, \gamma \in \Phi^{+}$, if $\gamma=a \alpha+b \beta, a>0, b>0$, then $\alpha \preceq \gamma \preceq \beta$, or $\beta \preceq \gamma \preceq \alpha$. It is known that, in the case of finite Coxeter groups, we have a one-to-one correspondence between reduced decompositions of the maximal 
element (i.e. maximal chains in the weak order) and reflection orderings. More precisely, if $W$ is finite, then a chain $\mathcal{C}$ of $\left(\mathcal{B}\left(\Phi^{+}\right), \subseteq\right)$ is maximal if and only if there exists a reflection ordering $I$ such that $\mathcal{C}$ is constituted exactly of the initial sections (i.e. lower sets) of $I$. The second conjecture of Dyer says that this situation is preserved in the infinite case. More precisely, the second conjecture of Dyer says that a chain $\mathcal{C}$ of $\left(\mathcal{B}\left(\Phi^{+}\right), \subseteq\right)$ is maximal if and only if there exists a reflection ordering $I$ such that $\mathcal{C}$ is constituted exactly of the initial sections of $I$. As one can see, the notion of peeling sequence (see Section 22 provides an equivalent of reflections orderings for lattices coming from finite acyclic valued digraph. However, projective valued digraphs are limit of finite acyclic valued digraph, hence it is natural to look for a generalization of the notion of peeling sequence to the context of projective valued digraph. Fortunately, such a generalization exists: we can associate each projective valued digraph $\mathcal{G}_{\infty}$ with a set of total orderings of the vertices of $\mathcal{G}_{\infty}$, which we denote by $\operatorname{PS}\left(\mathcal{G}_{\infty}\right)$, generalizing the notion of peeling sequences.

Definition 3.5 Let $\mathcal{G}_{\infty}$ be a projective valued digraph. A total ordering $I=\left(\mathcal{V}\left(G_{\infty}\right)\right.$, $)$ is called a peeling of $\mathcal{G}_{\infty}$ if and only if for all $z \in \mathcal{V}\left(G_{\infty}\right)$, we have $\theta(z)=\left|\left\{y \preceq z \mid(z, y) \in E\left(G_{\infty}\right)\right\}\right|$. We denote by $P S\left(\mathcal{G}_{\infty}\right)$ the set of all the peelings of $G$.

Remark 3.6 Before we move to the study of some properties of this notion of peeling, let us make two important remarks.

1. One can easily check by induction that this definition is equivalent to the definition of peeling sequences in the finite simple acyclic case.

2. This definition applies to any valued digraph, and one can associate any valued digraph with a set of peelings. However, in this very general context, peelings do not have many of the properties they have in the finite acyclic or in the projective case (for instance, Theorem 3.7 is false in general).

Theorem 3.7 Let $\mathcal{G}_{\infty}$ be a projective valued digraph and $\mathcal{C}$ be a chain of $\left(\operatorname{IS}\left(\mathcal{G}_{\infty}\right), \subseteq\right)$. Then, there exists $I \in P S\left(\mathcal{G}_{\infty}\right)$ such that $\mathcal{C}$ is included in the set of the initial sections of I (i.e. the lower sets of I), and $\mathcal{C}$ is maximal if and only if we have equality.

Proof: Since the proof of this theorem is quite involved, we refer the interested reader to [18, Section 4.2]. However, we give the key property: let us denote by $\left(G_{i}\right)_{i \geq 1}$ a sequence of finite acyclic valued digraphs such that $\mathcal{G}_{\infty}$ is the limit of this sequence. Thanks to Remark $3.6(1)$, for all $i<\infty$, the elements of $P S\left(\mathcal{G}_{i}\right)$ are the total orderings of $\mathcal{V}\left(G_{i}\right)$ satisfying Definition 3.5. Thus, for all $i<j \leq \infty$ and $J \in P S\left(\mathcal{G}_{j}\right)$, thanks to (1) we have that the total order $I$ of $\mathcal{V}\left(\mathcal{G}_{i}\right)$ obtained from $J$ by suppressing the elements of $\mathcal{V}\left(G_{j}\right) \backslash \mathcal{V}\left(G_{i}\right)$ and keeping the same order is an element of $P S\left(\mathcal{G}_{i}\right)$. In other words, the family $\left(P S\left(\mathcal{G}_{k}\right)\right)_{1 \leq k \leq \infty}$ is a projective system, and one can study the properties of of $P S\left(\mathcal{G}_{\infty}\right)$ using the properties of the peeling sequences in the finite acyclic case. In particular, many properties that are true for peeling sequences remain true for peelings in the projective case. This is the case here.

There is a clear similarity between the previous Theorem and the second conjecture of Dyer. Furthermore, as claimed earlier and shown in [18, Section 4.3], one can use well-assembled valued digraphs to construct extensions of the weak order containing $\left(\mathcal{B}\left(\Phi^{+}\right), \subseteq\right)$. However, well-assembled valued digraphs are made of scaffoldings glued together, and scaffoldings are clearly projective. Consequently, it is natural to ask if it is possible to construct a projective well-assembled valued digraph, and see if it leads to some information about the second conjecture of Dyer. Indeed, one can show that such a valued digraph exists 
and can be explicitly constructed. Unfortunately, this construction is quite involved and uses some refined properties of an usual statistic on the positive roots: the depth. Thus, we will not detail this here and we refer the reader to [18]. However, we summarize in the following theorem some important consequences of the existence of such a valued digraph.

Theorem 3.8 Let $W$ be a Coxeter group of finite rank, $\Phi=\Phi^{+} \sqcup \Phi^{\text {? }}$ be the associated root system, $\mathcal{B}\left(\Phi^{+}\right)$be its set of bi-closed sets and $\mathcal{G}^{\infty}$ be a projective well-assembled on $\Phi^{+}$valued digraph. Then, we have the following two properties.

1. The poset $\left(I S\left(\mathcal{G}_{\infty}\right), \subseteq\right)$ is a complete algebraic ortho-lattice such that $\mathcal{B}\left(\Phi^{+}\right) \subseteq I S\left(\mathcal{G}_{\infty}\right)$, and each reflection ordering of $\Phi^{+}$is in $P S\left(\mathcal{G}^{\infty}\right)$.

2. We have that $I S\left(\mathcal{G}^{\infty}\right)=\mathcal{B}\left(\Phi^{+}\right)$if and only if $P S\left(\mathcal{G}_{\infty}\right)$ is equal to the set of the reflection orderings of $\Phi^{+}$(i.e. the two conjectures of Dyer are equivalent in our context).

After many tests using the projective structure of projective well-assembled valued digraphs, we make the conjecture that there exists at least one projective well-assembled valued digraph $\mathcal{G}_{\infty}$ such that $I S\left(\mathcal{G}_{\infty}\right)=$ $\mathcal{B}\left(\Phi^{+}\right)$. Moreover, we prove that this conjecture holds for the Coxeter groups $A_{n}, B_{n}, D_{n}, E_{6}, E_{7}$ and $E_{8}$, generalizing the results presented in [17, Theo. 4.1].

\section{Description of the Tamari Lattice using valued digraphs}

Notice that the results mentioned in this section arise in a more general context, involving Cambrian semilattices. More precisely, one can use the concept of valued digraphs in order to construct extensions of Cambrian semi-lattices into complete lattices. However, at this stage there is no clear connections between these extensions and combinatorics of cluster algebras, and we are not able to prove that our construction coincide with the classical one even in type $A$ (despite many empirical evidences). Nevertheless, this can be done in the special case of Tamari lattice, using combinatorial techniques.

Definition 4.1 We denote by $\mathcal{A}_{n}^{\uparrow}=\left(G_{n}, \theta\right)$ the valued digraph such that:

$$
\begin{aligned}
\mathcal{V}\left(\mathcal{A}_{n}^{\uparrow}\right) & =\{(a, b) \mid 1 \leq a<b \leq n+1\}, \\
E\left(\mathcal{A}_{n}^{\uparrow}\right) & =\{((a, b),(a, d)) \mid b>d\} \cup\{((a, c),(b, c)) \mid a<b\} \cup\{((a, b),(a, d)) \mid b<d\}, \\
\theta(a, b) & =b-a-1 \text { for all }(a, b) \in \mathcal{V}\left(\mathcal{A}_{n}^{\uparrow}\right) .
\end{aligned}
$$

Note that the valued digraph $\mathcal{A}_{n}^{\uparrow}$ is in fact obtained from the valued digraph associated with the weak order on $A_{n}$ (see [17]) by adding some arcs to it, as depicted on Figure 5 .

On Figure 6, we represent the lattices associated with $\mathcal{A}_{2}^{\uparrow}$ and $\mathcal{A}_{3}^{\uparrow}$, where the valued digraphs are represented with staircase diagram (the structure of digraph is implicit) and where the red boxes correspond to the elements of $I S\left(\mathcal{A}_{n}^{\uparrow}\right)$. In the first case, we can recognize the Tamari lattice $\mathcal{T}_{3}$, and in the second $\mathcal{T}_{4}$. This situation is in fact general, and we can show the following theorem by constructing an explicit bijection between $I S\left(\mathcal{A}_{n}^{\uparrow}\right)$ and Dyck paths of size $n+1$, which turns out to be a poset isomorphism (see [18, Chapter 5] for a proof).

Theorem 4.2 The lattice $\left(I S\left(\mathcal{A}_{n}^{\uparrow}\right), \subseteq\right)$ is isomorphic to the $(n+1)$-th Tamari lattice $\mathcal{T}_{n+1}$. 


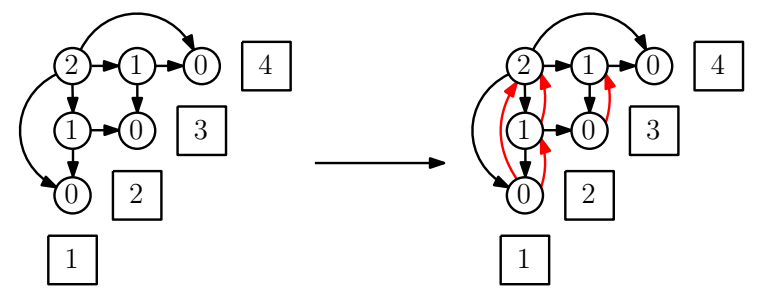

Fig. 5: From the valued digraph associated with the weak order on $A_{3}$ to $\mathcal{A}_{3}^{\uparrow}$.
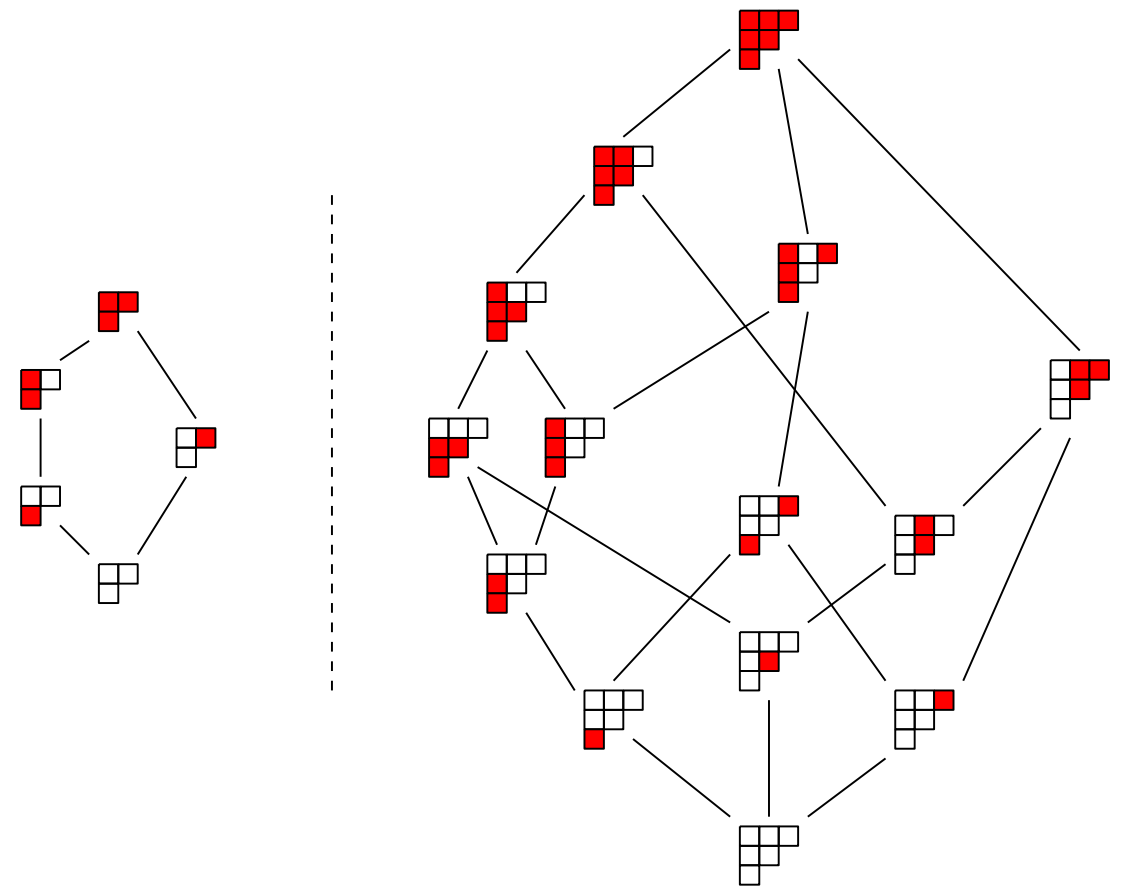

Fig. 6: The lattices $\left(I S\left(\mathcal{A}_{2}^{\uparrow}\right), \subseteq\right)$ and $\left(I S\left(\mathcal{A}_{3}^{\uparrow}\right), \subseteq\right)$.

\section{References}

[1] A. Bjorner, F. Brenti, Combinatorics of Coxeter groups. Springer, New York, NY, 2005.

[2] M. Dyer, Hecke algebras and shellings of Bruhat intervals. Compos. Math. 89, no. 1, 91-115, 1993.

[3] M. Dyer, On the weak order of Coxeter groups, Preprint arXiv.org:1108.5557, 2011.

[4] M. Dyer, Groupoids, root systems and weak order I. Preprint arXiv:1110.2317, 2011 (47 pages) 
[5] M. Dyer, Groupoids, root systems and weak order II. Preprint arXiv:1110.3657, 2011 (52 pages)

[6] M. Dyer, C. Hohlweg, V. Ripoll, Imaginary cones and limit roots of infinite Coxeter groups. Preprint arXiv.org:1303.6710, 2013.

[7] C. Hohlweg, J.-P. Labbé, On inversion sets and the weak order on Coxeter group. To appear in European J. of Combinatorics.

[8] C. Hohlweg, J.-P. Labbé, V. Ripoll, Asymptotical behaviour of roots of infinite Coxeter groups. Canad. J. Math. 66 (2014), no.2, 323-353.

[9] J.-P. Labbé, Polyhedral combinatorics of Coxeter group. PhD Thesis, Freie Universität, Berlin (2013).

[10] A. Pilkington, Convex geometries on root systems, Comm. Algebra 34, no.9, 3183-3202, 2006.

[11] N. Reading, Cambrian lattices. Adv. Math. 205 (2006), no.2 313-353.

[12] N. Reading, Clusters, Coxeter-sortable elements and noncrossing partitions. Trans. Amer. Math. Soc. 359 (2007), no.12 5931-5958.

[13] N. Reading, Sortable elements and Cambrian lattices. Algebra Universalis 56 (2007), no.3-4 411437.

[14] N. Reading, D. E Speyer, Sortable elements in infinite Coxeter groups. Trans. Amer. Math. Soc. 363 (2007), no.2 699-761.

[15] N. Reading, D. E Speyer, Combinatorial frameworks for cluster algebras. Int. Math. Res. Notices (2016), no.1 109-173.

[16] N. Reading, D. E Speyer, Cambrians frameworks for cluster algebras of affine type. Preprint arXiv:1504.00260, 2011 (40 pages).

[17] F. Viard, A new family of posets generalizing the weak order on some Coxeter groups. Preprint arXiv:1504.00260, 2011 (40 pages).

[18] F. Viard, From valued digraphs to complete lattices: a new approach of the weak order on Coxeter groups. PhD Thesis, Université Claude Bernard. Available at http://math.univ-lyon1.fr/homeswww/viard/These.pdf 